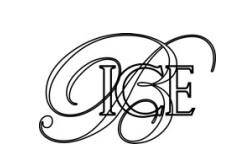

\title{
LA CRECIENTE IMPORTANCIA DEL «ESPAÑOL PARA LOS NEGOCIOS» EN LAS INSTITUCIONES DE ENSEÑANZA SUPERIOR DE EEUU
}

La creciente importancia del español como lenguaje de los negocios en Estados Unidos ha propiciado que las instituciones de enseñanza superior estadounidenses hayan comenzado a ofrecer asignaturas y titulaciones que combinan el estudio del español y los temas empresariales.

El presente artículo analiza el panorama actual de la enseñanza del Español para los Negocios en EEUU tomando como muestra las 100 mejores escuelas de negocio/facultades de empresariales, según el ranking de 2014 publicado por Bloomberg. Como conclusión, puede afirmarse que todas las instituciones consultadas ofrecen la posibilidad de combinar el estudio del español y los temas empresariales aunque los niveles de integración de ambas materias varían de forma significativa. Así, en un primer nivel (14 por 100 de la muestra) se encontrarían las instituciones que aún no han desarrollado cursos específicos que aborden el estudio de temas empresariales en español aunque es posible estudiar ambas materias por separado. El segundo nivel engloba a las 52 facultades que ofrecen cursos de español para los negocios y, finalmente, en el tercer nivel se encuentran los 34 centros que ofertan titulaciones de grado, posgrado y formación ejecutiva especialmente diseñadas para los estudiantes que deseen desarrollar su actividad profesional en empresas o países de habla hispana.

A continuación, y con el fin de promover la mejora en la enseñanza de esta materia, el artículo identifica las mejores estrategias seguidas por las escuelas de negocio consultadas. Entre ellas destacan la formación práctica, las estancias internacionales, la flexibilidad curricular, el enfoque multidisciplinar, el uso de las tecnologías de la información y comunicación y la multiculturalidad.

Palabras clave: escuelas de negocio, enseñanza universitaria, empresas españolas, crecimiento empresarial. Clasificación JEL: A22, I23.

\section{Introducción}

El número de hispanohablantes en Estados Unidos (EEUU) se ha incrementado de forma gradual durante las últimas décadas. Según el Pew Research Center ${ }^{1}$, en 2013, el número de hispanos

\footnotetext{
* Department of Business Administration. Stonehill College. Versión de julio de 2015.

1 El Pew Research Center es un centro de investigación, con sede en Washington DC, que analiza y suministra información sobre problemáticas sociales, opinión pública y tendencias demográficas que afectan a los Estados Unidos y al mundo.
}

en EEUU era de 54 millones, equivalente al 17,1 por 100 de la población, lo que supone un aumento significativo si se compara con los 14,8 millones que componían tan sólo el 6,5 por 100 de la población americana en 1980.

Este notable incremento de la población hispana ha venido acompañado de un espectacular crecimiento en su capacidad adquisitiva. Jiménez y Narbona (2012) analizaron la evolución del poder de compra de los hispanos, llegando a la conclusión de que éste ha sido el más alto entre los $\triangleright$ 
grupos minoritarios de EEUU desde 2007. En este sentido, el Centro de Investigación Selig, de la Universidad de Georgia, afirma que el mercado hispano de 1,3 billones de dólares en 2014 registró un aumento de poder adquisitivo del 155 por 100 desde 2000, significativamente superior al 71 por 100 de la población no hispana y el 76 por 100 de la población total (Selig Center for Economic Growth, 2014). De este modo, podría concluirse que la población hispana se configura como el mercado con mayor crecimiento en EEUU. A pesar de que el hecho de ser hispano no implica un conocimiento efectivo del español, la correlación entre ambas variables es muy elevada ${ }^{2}$. Por tanto, es posible afirmar que el español se está convirtiendo en un lenguaje esencial para desenvolverse en el mundo empresarial de EEUU.

Las instituciones de enseñanza superior estadounidenses son conscientes de esta realidad y, fieles a su misión de proporcionar a sus alumnos los conocimientos y habilidades necesarios para triunfar en el entorno actual, han comenzado a ofrecer asignaturas y programas que combinan el lenguaje español y los temas empresariales. Así, los estudiantes serán capaces de desarrollar las capacidades lingüísticas necesarias para desenvolverse en el mundo empresarial de ámbito hispano.

Este hecho pone de manifiesto la fuerte interrelación que existe entre las lenguas y la actividad económica. Por un lado, el lenguaje actúa como facilitador de las transacciones económicas ya que el uso de un idioma común resulta esencial para que las distintas partes implicadas comprendan todos sus aspectos y puedan llevarlas a buen término. A su vez, las transacciones económicas en una lengua extranjera pueden favorecer la expansión de dicha lengua en un determinado país. Por tanto, es necesario contar con cursos especializados y de calidad que proporcionen los conocimientos y herramientas necesarios para conseguir el dominio del

\footnotetext{
2 La Oficina del Censo de EEUU (2010) concluye que más del 73 por 100 de las familias hispanas utilizan en mayor o menor medida el español para comunicarse y únicamente el 26,7 por 100 usa solo el inglés.
}

español en cualquier situación laboral y mercantil. Para alcanzar este objetivo, sería más que recomendable aprender de las buenas prácticas que actualmente se están llevando a cabo en las instituciones de enseñanza superior de EEUU.

En el presente artículo se ofrece un análisis detallado y eminentemente descriptivo del panorama actual de la enseñanza del Español para los Negocios en EEUU para, posteriormente, identificar aquellas estrategias que permiten un aprendizaje más efectivo del mismo. Pero, antes de abordar esta cuestión, se analizará brevemente el valor económico del español y el papel fundamental que éste desempeña como facilitador de la actividad económica.

\section{El español como soporte y facilitador de la actividad económica}

En el mundo globalizado en el que vivimos, la actividad económica y las lenguas están fuertemente interrelacionadas. Por un lado, el lenguaje es el principal instrumento de comunicación entre los seres humanos y resulta imprescindible a la hora de realizar cualquier transacción económica. El español, hablado por más de 540 millones de personas como lengua nativa, es el segundo idioma de comunicación internacional (Instituto Cervantes, 2014) y este hecho resulta altamente beneficioso para las empresas españolas. Si circunscribimos nuestro análisis a EEUU, podemos observar (Tabla 1) como el volumen de negocio de las filiales de empresas españolas en este país ha ido creciendo de forma continua.

Un análisis más exhaustivo de las empresas españolas establecidas en los estados del noreste de EEUU (Tabla 2) pone de manifiesto la influencia de sectores tan emblemáticos como la moda, los productos de alimentación y los servicios financieros en la economía de esta región estadounidense.

Ahora bien, no hemos de olvidar que las empresas españolas son sólo uno de los responsables del incremento de la actividad económica $\triangle$ 


\begin{tabular}{|c|c|c|c|}
\hline \multicolumn{4}{|c|}{$\begin{array}{c}\text { TABLA } 1 \\
\text { ESTADÍSTICA DE FILIALES DE EMPRESAS ESPAÑOLAS EN EEUU }\end{array}$} \\
\hline Año & Numero de filiales & Personas ocupadas & $\begin{array}{l}\text { Volumen de negocio } \\
\text { (miles de euros) }\end{array}$ \\
\hline 2009 & 341 & 36.073 & 13.692 .685 \\
\hline 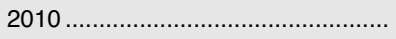 & 295 & 31.750 & 14.535 .238 \\
\hline 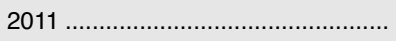 & 305 & 31.059 & 18.159 .732 \\
\hline 2012 & 287 & 31.538 & 20.410 .015 \\
\hline
\end{tabular}

\begin{tabular}{|c|c|c|c|}
\hline \multicolumn{4}{|c|}{$\begin{array}{c}\text { TABLA } 2 \\
\text { EMPRESAS ESPAÑOLAS ESTABLECIDAS EN EEUU; ESTADOS DEL NORESTE (MARZO 2014) }\end{array}$} \\
\hline Sectores & Subsectores & $\mathbf{N}^{\circ}$ empresas & Porcentaje \\
\hline Sector agroalimentario & $\begin{array}{l}\text { Grasas y aceites } \\
\text { Panadería y otros } \\
\text { Pescados y mariscos } \\
\text { Productos cárnicos } \\
\text { Productos hortofrutícolas }\end{array}$ & $\begin{array}{l}5 \\
2 \\
1 \\
2 \\
7\end{array}$ & $\begin{array}{l}3,11 \\
1,24 \\
0,62 \\
1,24 \\
4,35\end{array}$ \\
\hline \multicolumn{2}{|c|}{ Total sector agroalimentario... } & 17 & 10,56 \\
\hline Bebidas & 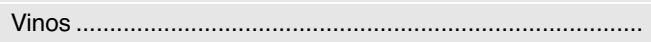 & 7 & 4,35 \\
\hline \multicolumn{2}{|c|}{ Total sector bebidas } & 7 & 4,35 \\
\hline Bienes de consumo & 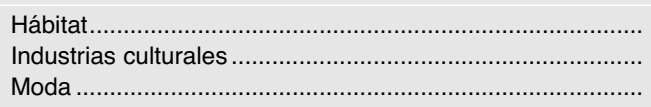 & $\begin{array}{r}13 \\
3 \\
25\end{array}$ & $\begin{array}{r}8,07 \\
1,86 \\
15,53\end{array}$ \\
\hline \multicolumn{2}{|c|}{ Total sector bienes de consumo } & 41 & 25,47 \\
\hline $\begin{array}{l}\text { Industria y tecnología } \\
\text { (Total: } 43 \text { empresas) }\end{array}$ & 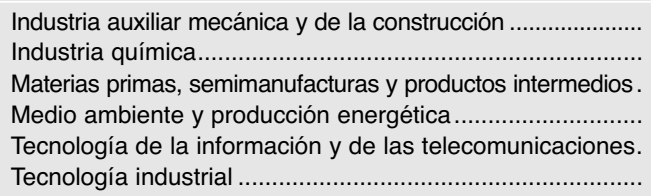 & $\begin{array}{r}6 \\
4 \\
3 \\
11 \\
5 \\
14\end{array}$ & $\begin{array}{r}3,7 \\
2,48 \\
1,86 \\
6,83 \\
3,11 \\
8,70\end{array}$ \\
\hline \multicolumn{2}{|c|}{ Total sector industria y tecnología } & 43 & 26,71 \\
\hline Servicios & $\begin{array}{l}\text { Infraestructuras, construcción civil e ingeniería. } \\
\text { Servicios a empresas. } \\
\text { Servicios de industrias culturales } \\
\text { Servicios de tecnología industrial } \\
\text { Transporte, logística, y comunicaciones } \\
\text { Turismo e inmobiliario } \\
\text { Otros servicios comerciales y mercantiles }\end{array}$ & $\begin{array}{r}1 \\
17 \\
2 \\
1 \\
19 \\
11 \\
2\end{array}$ & $\begin{array}{r}0,62 \\
10,56 \\
1,24 \\
0,62 \\
11,80 \\
6,83 \\
1,24\end{array}$ \\
\hline \multicolumn{2}{|c|}{ Total sector servicios } & 53 & 32,92 \\
\hline \multicolumn{2}{|c|}{ Total sectores analizados } & 161 & 100,00 \\
\hline
\end{tabular}

en español en EEUU. El otro gran protagonista sería el sector empresarial latino que, según el informe anual 2014 publicado por Geospace en colaboración con la Cámara Hispana de Comercio de los Estados Unidos (Geospace, 2014), ha crecido de forma exponencial durante la última década.

Según este estudio, el sector empresarial hispano aumentó un 43 por 100 desde 2007 hasta 2014, lo que supone el doble de la tasa de crecimiento experimentado por el conjunto de empresas de la economía estadounidense (20,2 por 100). Asimismo, se pone de manifiesto el relevante y progresivo impacto del sector latino en la economía de EEUU mediante el pago de impuestos, la creación de puestos de trabajo, y la generación de riqueza. Entre las razones que, según este informe, explican este dinamismo empresarial se encontrarían la juventud y el carácter emprendedor de la población de origen hispano que, bien por iniciativa propia o por las dificultades a las que se enfrentan para acceder a otras oportunidades laborales, deciden crear su propio negocio.

Una vez demostrado cómo el crecimiento de la actividad comercial de empresas hispanohablantes $\triangleright$ 
en EEUU está favoreciendo la paulatina expansión del español como lenguaje de los negocios en este país, se comprende la necesidad de contar con una correcta enseñanza del mismo. Introducimos así el concepto de lengua como mercado, concepto que se refiere a la enseñanza del idioma y las actividades mercantiles asociadas a ella.

El número de personas que estudian español como segunda lengua está experimentando un fuerte crecimiento y el Instituto Cervantes (2014) estima que existen 7.820 .000 estudiantes de español en EEUU. Para reforzar esta tendencia, es necesario contar con cursos especializados y de calidad que proporcionen los conocimientos y herramientas necesarios para conseguir el dominio del español en cualquier situación laboral y mercantil. Este objetivo no puede conseguirse mediante fórmulas improvisadas; es necesario analizar el estado de la cuestión para así poder identificar un conjunto de buenas prácticas que permitan un aprendizaje más efectivo y, en consecuencia, una mayor expansión del español como idioma empresarial a nivel mundial.

\section{Enseñanza del Español para los Negocios en EEUU. Análisis de la situación actual}

\subsection{Tamaño y composición de la muestra}

Para analizar el panorama actual de la enseñanza del Español para los Negocios en EEUU se han tomado como muestra las 100 mejores escuelas de negocio/facultades de empresariales según el ranking de 2014 publicado por Bloomberg ${ }^{3}$.

Un estudio inicial de la muestra permite comprobar que todas las divisiones territoriales que componen las cuatro regiones de Estados Unidos están representadas (Tabla 3). Asimismo, la muestra incluye instituciones de distintas dimensiones, desde las más pequeñas, con menos de 500 alumnos

\footnotetext{
${ }^{3}$ El Anexo A ofrece una lista detallada de las instituciones que componen la muestra.
}

hasta aquellas de mayor escala, con más de 5.000 estudiantes (Gráfico 1).

\subsection{Situación actual del Español para los Negocios en EEUU}

Tras analizar la información proporcionada en sus páginas web por todas y cada una de las instituciones de la muestra, se puede concluir que todas ellas, de una u otra forma, ofrecen la posibilidad de combinar el estudio de la lengua española y los temas empresariales. Ahora bien, el nivel de integración entre ambas materias varía significativamente según el caso considerado. Así, en un extremo del espectro podríamos encontrar instituciones, como el Instituto Politécnico Rensselaer, que no cuentan con un departamento de lenguas pero que animan a sus alumnos a mejorar sus capacidades lingüísticas a través de acuerdos con instituciones que ofrecen formación en idiomas. En el otro extremo del espectro nos encontraríamos con centros como la Universidad de Miami que ofrece un amplio catálogo de programas y cursos de negocios en español, abarcando tanto los niveles de grado como los de posgrado y formación ejecutiva. Entre estos dos extremos, se sitúan las distintas opciones ofertadas por las instituciones de la muestra y que, tras un concienzudo análisis, se han podido clasificar en tres niveles (Esquema 1).

\section{Nivel 1: No se ofrece el curso «Español para los Negocios"}

En un primer nivel (14 por 100 de la muestra), encontraríamos aquellas instituciones que ofrecen, de forma separada, cursos de español y de negocios pero que todavía no han desarrollado cursos que combinen ambas materias. En este caso, la opción disponible para aquellos alumnos que deseen desarrollar su actividad profesional futura en español seria elegir una doble titulación, preferiblemente un grado en negocios internacionales y un grado o especialización en español. 


\section{LA CRECIENTE IMPORTANCIA DEL «ESPAÑOL PARA LOS NEGOCIOS»...}

\begin{tabular}{|c|c|c|c|c|c|c|c|c|}
\hline \multicolumn{9}{|c|}{$\begin{array}{c}\text { TABLA } 3 \\
\text { PROCEDENCIA DE LAS INSTITUCIONES DE ENSEÑANZA SUPERIOR ANALIZADAS }\end{array}$} \\
\hline \multicolumn{2}{|c|}{ Región 1: Noreste } & \multicolumn{2}{|c|}{ Región 2: Medio Oeste } & \multicolumn{3}{|c|}{ Región 3: Sur } & \multicolumn{2}{|c|}{ Región 4: Oeste } \\
\hline División 1 & División 2 & División 3 & División 4 & División 5 & División 6 & División 7 & División 8 & División 9 \\
\hline New England & Mid-Atlantic & $\begin{array}{l}\text { East North } \\
\text { Central }\end{array}$ & $\begin{array}{l}\text { West North } \\
\text { Central }\end{array}$ & South Atlantic & $\begin{array}{l}\text { East South } \\
\text { Central }\end{array}$ & $\begin{array}{l}\text { West South } \\
\text { Central }\end{array}$ & Mountain & Pacific \\
\hline 12 & 16 & 19 & 5 & 21 & 2 & 10 & 6 & 9 \\
\hline \multicolumn{2}{|c|}{28 Instituciones } & \multicolumn{2}{|c|}{24 Instituciones } & \multicolumn{3}{|c|}{33 Instituciones } & \multicolumn{2}{|c|}{15 Instituciones } \\
\hline \multicolumn{9}{|c|}{ Muestra total: 100 Facultades de Empresariales/ Escuelas de Negocios } \\
\hline \multicolumn{9}{|c|}{$\begin{array}{l}\text { Notas: } \\
\text { División } 1 \text { (New England): Connecticut, Maine, Massachusetts, New Hampshire, Rhode Island, Ver } \\
\text { División } 2 \text { (Mid Atlantic): New Jersey, New York, Pennsylvania. } \\
\text { División } 3 \text { (East North Central): Illionois, Indiana, Michigan, Ohio, Wisconsin. } \\
\text { División } 4 \text { (West North Central): Iowa, Kansas, Minnesota, Missouri, Nebraska, North Dakota, Sout } \\
\text { División } 5 \text { (South Atlantic): Delware, Florida, Georgia, Maryland, North Carolina, South Carolina, Vir } \\
\text { División } 6 \text { (East South Central): Alabama, Kentucky, Mississippi, Tennessee. } \\
\text { División } 7 \text { (West South Central): Arkansas, Louisiana, Oklahoma, Texas. } \\
\text { División } 8 \text { (Mountain): Arizona, Colorado, Idaho, Montana, Nevada, New Mexico, Utah, Wyoming. } \\
\text { División } 9 \text { (Pacific): Alaska, California, Hawaii, Oregón, Washington. } \\
\text { Fuente: elaboración propia. }\end{array}$} \\
\hline
\end{tabular}

\section{GRÁFICO 1}

TAMAÑO DE LAS INSTITUCIONES DE ENSEÑANZA SUPERIOR ANALIZADAS

(Número de alumnos matriculados en los distintos programas de negocios)

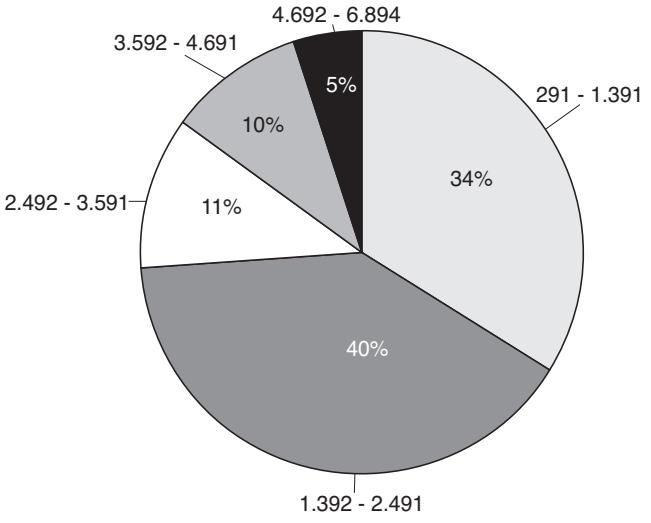

Fuente: elaboración propia.

ESQUEMA 1

NIVELES DE ENSEÑANZA DEL ESPAÑOL PARA LOS NEGOCIOS

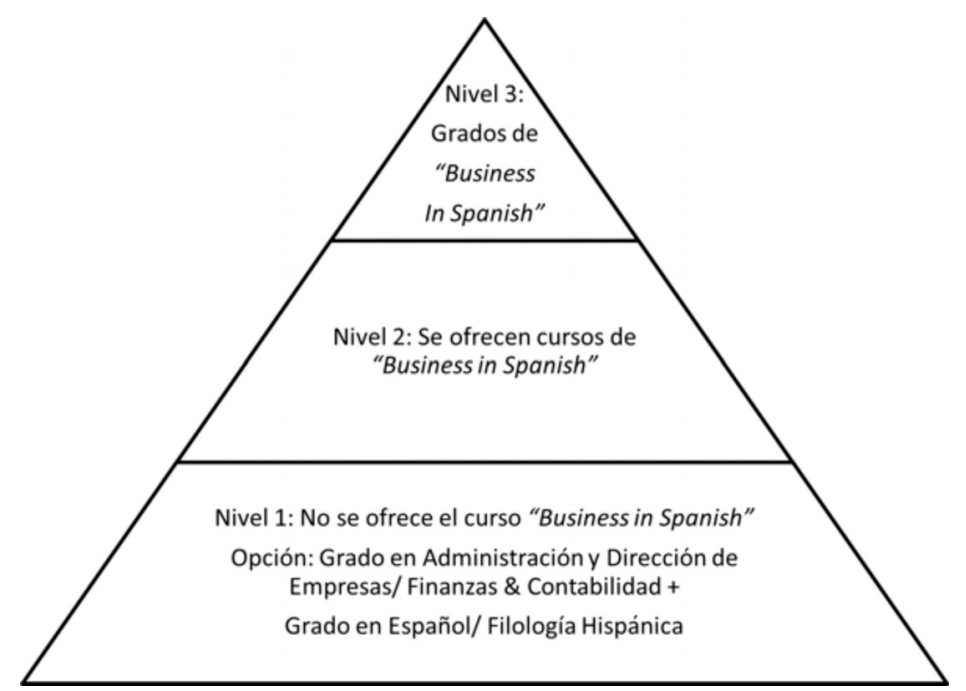

Fuente: elaboración propia. 
Nivel 2: Se ofrecen cursos específicos sobre «Español para los Negocios»

En un segundo nivel se sitúan aquellas instituciones que ofrecen asignaturas sobre temas empresariales en español. Este sería el caso de 52 de los centros analizados que, bajo distintas denominaciones, incluyen este tipo de cursos en sus catálogos. En la mayoría de ocasiones (90 por 100 de la muestra) estas asignaturas se estudian a través de los departamentos de idiomas siendo minoritarias (10 por 100) las instituciones que ofrecen este tipo de cursos en sus facultades de empresariales. Una tercera opción, muy recomendable aunque observada tan solo en una de las universidades de la muestra, sería la de la referencia cruzada; en este caso, los dos departamentos incluirían el curso bajo un doble prefijo dejando patente la interrelación entre ambas materias.

Los títulos que se emplean con mayor frecuencia son: Spanish for Business (Español para los Negocios), Business Spanish (Negocios en Español), Commercial Spanish (Español Comercial) y Spanish for professional purposes: business (Español para fines profesionales: negocios). También destacan aquellos cursos que se centran más específicamente en la vertiente comunicativa del lenguaje, tanto oral como escrito, con títulos como Business Communication and Correspondence (Comunicación y correspondencia empresarial) o Business Writing in Spanish (Escritura de temas empresariales en Español). Otros cursos focalizan más su atención en los aspectos culturales como es el caso de Language and Culture in Business (Lenguaje y cultura empresarial), The Economy and Politics of Latin Americ (La economía y política en Latinoamérica), o Latin American culture in a business context (Cultura latinoamericana en un contexto empresarial). Especial atención merecen aquellos cursos que, mediante proyectos basados en empresas reales que operan en español, y que proceden de España o Sudamérica, permiten a los alumnos poner en práctica todo lo aprendido. A menudo estos cursos incluyen una estancia en el país de origen de las empresas estudiadas como ocurre con International Consulting: Latin America (Consultoría internacional: Latinoamérica), Spain \& Portugal start-up strategy (España y Portugal: estrategias de creación de empresas) y Chile \& Peru: Analyzing entrepreneurial opportunities in Latin America (Chile y Perú: analizando oportunidades para emprender nuevos negocios en Latinoamérica). Siguiendo esta misma línea, encontraríamos cursos que tratan de fomentar la innovación y el espíritu emprendedor así como la sostenibilidad y la responsabilidad corporativa tales como Spanish and Entrepreneurship (Español y espíritu emprendedor), Spanish for Social Innovation (Español para la innovación social), Sustainability and Corporate Social Responsibility in Costa Rica (Sostenibilidad, y responsabilidad social corporativa en Costa Rica).

\section{Nivel 3: Se ofrecen titulaciones específicas de «Español para los Negocios»}

En el tercer y último nivel encontraríamos los 34 centros de enseñanza superior que ofrecen titulaciones específicas de Español para los Negocios. Las opciones de esta categoría son muy variadas, desde especialidades de Español para los Negocios (Business Spanish Minor-titulación secundaria de español-, Certificate in Spanish Studies for Business Majors -certificado de español para estudiantes de empresariales-) ofrecidas por los departamentos de idiomas, hasta títulos de grado y posgrado incluidos en los catálogos de las facultades de empresariales y escuelas de negocios. Dentro de este último grupo son frecuentes las titulaciones en negocios internacionales, con distintos itinerarios lingüísticos, que se ajustan a los intereses específicos de los alumnos. De este modo, cada uno de ellos podría construir su propio plan de estudios escogiendo aquellas asignaturas que abordan tanto los aspectos lingüísticos como culturales de la zona en la que quieren desarrollar su futura carrera profesional. Como ejemplo de este tipo de programas se podría citar a la Marriott School of Management (Brigham Young University) que, en su afán de ayudar a sus alumnos a triunfar en $\triangleright$ 


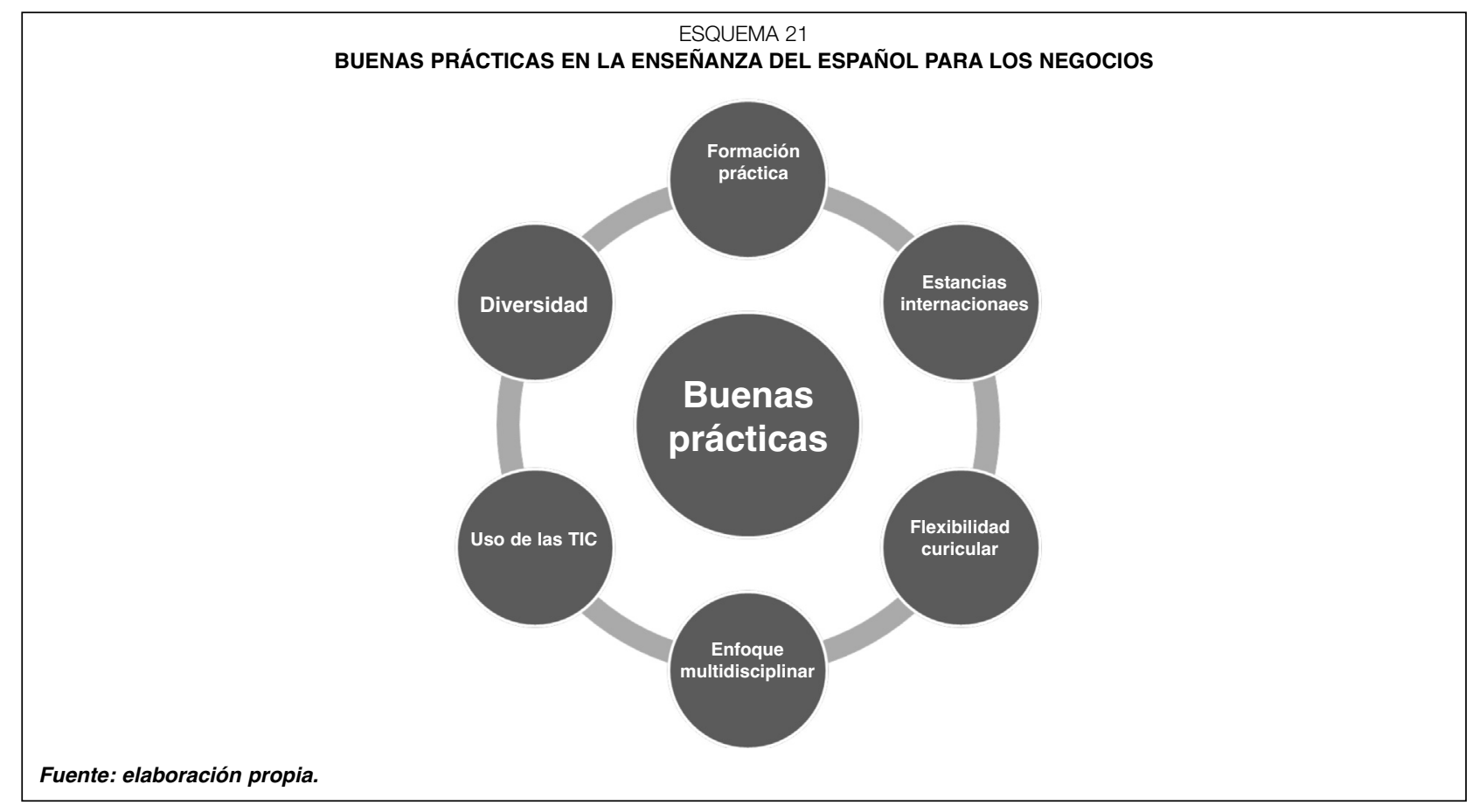

el ámbito internacional, ofrece la posibilidad de elegir entre un total de diez idiomas (español, árabe, chino, francés, alemán, italiano, japonés, coreano, portugués y ruso).

La colaboración es una práctica muy extendida a este nivel. Así, encontramos programas ofrecidos conjuntamente por los departamentos de idiomas y las facultades de empresariales/escuelas de negocios y que permiten obtener un doble master en temas empresariales y lingüísticos o internacionales. En muchas ocasiones, la colaboración sobrepasa las fronteras de la propia universidad e incluso del país, y son frecuentes los acuerdos con instituciones educativas de reconocido prestigio de otros países, preferentemente España y Sudamérica. Como ejemplos podrían citarse la Universidad de Georgetown que mantiene acuerdos con ESADE (Escuela Superior de Administración y Dirección de Empresas) y la Universidad de Northeastern, que tiene una fuerte vinculación con ICADE (Instituto Católico de Administración y Dirección de Empresas). También resulta interesante el caso de la Universidad de Sant Louis, que en la actualidad cuenta con un campus propio en Madrid en el que estudiantes de más de 65 países pueden cursar estudios de grado y posgrado.

\section{Buenas prácticas en la enseñanza del Español para los Negocios}

Un análisis exhaustivo de las 100 mejores escuelas de negocios/facultades de empresariales de EEUU ha permitido identificar una gran multitud de buenas prácticas de las que pueden extraerse valiosas conclusiones para mejorar el proceso de enseñanza-aprendizaje del Español para los Negocios. Estas conclusiones pueden agruparse en torno a los siguientes temas (Esquema 2):

- Formación práctica: la puesta en práctica de los conocimientos teóricos es esencial en todo proceso de aprendizaje en general y en el de temas empresariales en particular. Las universidades analizadas, conscientes de esta realidad, ofrecen una gran variedad de oportunidades para que sus alumnos adquieran y ejerciten las habilidades que un buen directivo ha de tener, desde la utilización de programas informáticos que permiten realizar simulaciones con datos reales, hasta periodos de prácticas en empresas y en las ONG de habla hispana.

- Estancias internacionales: una de las mejores maneras de aprender y mejorar un idioma es viajando, ya que el hecho de vivir en un país donde $\triangleright$ 
se habla otra lengua obliga a practicarla constantemente. Pero además de mejorar los conocimientos lingüísticos, las estancias en el extranjero son muy beneficiosas ya que permiten al alumno aprender de otras culturas, madurar y fortalecer sus habilidades sociales, ampliando así sus oportunidades laborales.

Para maximizar el impacto positivo de estas experiencias, resulta fundamental establecer una tupida red de contactos internacionales, con acuerdos con escuelas de negocios de reconocido prestigio en países de habla hispana. Las Oficinas de Relaciones Internacionales así como las distintas asociaciones que promueven la diversidad y multiculturalidad en los centros de enseñanza superior estadounidenses se configuran como un recurso de incalculable valor a la hora de establecer y mantener estos acuerdos internacionales.

La combinación de formación práctica y estancias internacionales da lugar a cursos tan interesantes como los ofrecidos por Boston College y Texas Christian University (International Consulting: Latin America, Spain \& Portugal start-up strategy y Chile \& Peru: Analyzing entrepreneurial opportunities). Estos cursos, especialmente dirigidos a los alumnos que quieran dedicarse a la consultoría internacional, están divididos en dos partes bien diferenciadas. En una primera etapa, desarrollada en EEUU, los estudiantes llevan a cabo todas las tareas de investigación necesarias para elaborar un informe con recomendaciones específicamente diseñadas para una empresa latinoamericana con la que se comunican a través del teléfono, fax, email, y videoconferencias. En una segunda etapa, los distintos equipos viajan a los países de origen de las empresas con las que están trabajando para realizar las presentaciones personalmente y ofrecer un asesoramiento directo a sus clientes.

- Flexibilidad curricular: una de las principales características de las universidades estadounidenses es la flexibilidad. En la mayoría de ellas existe lo que se conoce como programa básico, que engloba los conocimientos fundamentales que todos los alumnos deben adquirir y, una vez satisfechos estos requisitos, cada estudiante tiene libertad para elegir entre un amplio abanico de grados y especializaciones, atendiendo a sus gustos y al futuro profesional que quiere tener. De hecho, las combinaciones son tan numerosas que puede resultar difícil encontrar dos personas que hayan cursado las mismas asignaturas.

Manteniendo este espíritu, sería deseable promover la flexibilidad en la enseñanza del Español para los Negocios a través de una oferta variada de cursos, que fuera más allá del tradicional Business Spanish, y abarcara otros aspectos como la cultura, la política y la economía de las regiones de habla hispana. Asimismo, sería beneficioso ofrecer distintos niveles de formación que puedan ajustarse a las necesidades específicas de los alumnos. Como ejemplo podría citarse la Universidad de Miami, que cuenta con una titulación de grado, Global Business Studies, que incluye cursos en español, dos programas master, The Miami Executive MBA for the Americas, Global Executive MBA (In Spanish), y multitud de programas de formación ejecutiva, destinados a profesionales, e impartidos en español e inglés, con traducción simultánea.

- Enfoque multidisciplinar: partiendo de la idea de que cada persona aprende de una forma distinta, es aconsejable diseñar los cursos utilizando una amplia variedad de estrategias y actividades que se adapten al estilo de aprendizaje especifico del mayor número posible de alumnos. Es necesario encontrar un equilibrio entre las actividades destinadas a transmitir contenidos y las que se centran en su aplicación y discusión.

Los centros educativos analizados aplican este enfoque multidisciplinar de forma consistente, y combinan todo tipo de actividades, desde las tradicionalmente desarrolladas en el aula hasta otras más novedosas como simulaciones por ordenador, proyectos de consultoría (que, como las comentadas anteriormente, suelen incluir viajes a los países de origen de dichas empresas), prácticas en empresas, actividades de voluntariado en las ONG de habla hispana, y estancias en universidades de reconocido prestigio de España y Sudamérica. También son $\triangleright$ 
muchas las instituciones que se benefician de la colaboración con otros departamentos, oficinas de relaciones internacionales y asociaciones/clubs de estudiantes que promueven la diversidad en sus distintas facetas.

- Uso de las tecnologías de la información y comunicación (TIC): las condiciones económicas cambian constantemente y hemos de ser conscientes de que no existen recetas ni fórmulas mágicas que puedan aplicarse para resolver de forma inequívoca los problemas empresariales. Por tanto, debemos ser capaces de enseñar a nuestros alumnos a aprender, es decir, hemos de facilitarles las herramientas que necesitan para identificar y analizar la información relevante en cada tipo de decisión. En este aprendizaje autónomo, que resulta fundamental en un entorno tan dinámico como el actual, las TIC pueden resultar de gran ayuda. Internet ofrece miles de posibilidades para aprender de forma independiente y puede resultar útil a la hora de perfeccionar un idioma. Además, las denominadas redes sociales pueden facilitar las interacciones necesarias para conseguir fluidez en las conversaciones, tanto orales como por escrito. Por ello sería deseable incorporarlas como un valioso recurso que permita complementar la formación presencial en los cursos de Español para los Negocios.

- Multiculturalidad: diversidad de profesores y alumnos. Son numerosos los estudios que han puesto de manifiesto el impacto positivo de la diversidad y multiculturalidad en los centros educativos (Gurin, 2004; Kitchener, et al., 2000; Pascarella et al., 2001). Estos autores demuestran que los estudiantes con una mayor exposición a las diferentes dimensiones de la diversidad, a través de interacciones con alumnos de distintas razas o participando en cursos y actos multiculturales en el propio campus, son los que más desarrollan su capacidad para pensar de forma analítica, reflexiva y crítica.

En esta misma línea, Thomson y Cuseo (2014) afirman que se debe promover la diversidad en las instituciones educativas porque aumenta el conocimiento y mejora el desarrollo personal y social de los alumnos, preparándoles para la sociedad global en la que vivimos y aumentando sus posibilidades de éxito profesional. Asimismo, estos autores argumentan que la diversidad promueve la creatividad al exponer las distintas perspectivas de un mismo problema. El impacto positivo de la diversidad en la innovación y la creatividad tampoco pasa desapercibido en el mundo profesional. En un estudio desarrollado por Forbes, el 85 por 100 de los directivos encuestados afirmaron que la diversidad es esencial para sus negocios mientras que el 75 por 100 declaró que dedicarían un mayor esfuerzo a promover la diversidad debido al papel que puede jugar en la consecución de sus objetivos empresariales (Forbes Insights, 2011).

Los beneficios que la diversidad aporta en las instituciones educativas se intensifican aún más en el caso de los alumnos que estudian materias con una elevada carga cultural como ocurre con el Español para los Negocios. La interacción de los estudiantes de estos cursos con profesores y compañeros internacionales, especialmente de países de habla hispana, permitiría el perfeccionamiento de sus habilidades lingüísticas a través de multitud de actividades que podrían organizarse. Entre estas actividades destaca, por su capacidad de promover la conversación espontánea y el desarrollo de la comprensión auditiva, la que se conoce como «tándem» o «intercambio de idiomas», que consiste en «emparejar» a dos personas las cuales dominan la lengua que la otra persona quiere aprender.

\section{Conclusiones}

La importancia del español como idioma de los negocios en EEUU ha ido creciendo de forma paulatina durante las últimas décadas. Este hecho se debe, no solo al incremento de la población hispana en este país sino también al aumento de las empresas de origen latino, que ha sido el doble del experimentado por el conjunto de empresas estadounidenses en el periodo 2007-2014 (Geoscape, 2014). 


\begin{tabular}{|c|c|c|c|c|c|}
\hline \multicolumn{6}{|c|}{$\begin{array}{l}\text { TABLA } 4 \\
\text { ESTADOS CON MAYOR PROPORCIÓN DE POBLACIÓN HISPANA - RESIDENTE } \\
\text { (En porcentaje) }\end{array}$} \\
\hline & 1980 & 1990 & 2000 & 2010 & 2013 \\
\hline 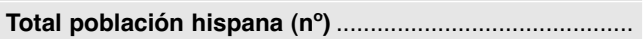 & 14.775 .080 & 21.838 .851 & 35.204 .480 & 50.729 .570 & 53.964 .235 \\
\hline Oeste & 42,70 & 45,50 & 43,40 & 40,80 & 40,30 \\
\hline California & 31,00 & 34,60 & 31,00 & 27,80 & 27,30 \\
\hline Sur & 30,60 & 30,40 & 32,80 & 36,10 & 36,60 \\
\hline 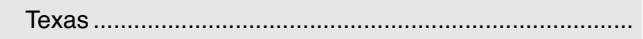 & 20,40 & 19,60 & 18,90 & 18,8 & 18,80 \\
\hline Florida & 5,90 & 7,10 & 7,60 & 8,40 & 8,60 \\
\hline Noreste & 17,90 & 16,60 & 14,90 & 13,80 & 14,00 \\
\hline 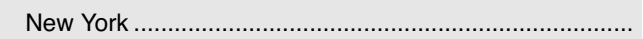 & 11,40 & 9,80 & 8,10 & 6,80 & 6,70 \\
\hline Medio Oeste & 8,70 & 7,60 & 8,90 & 9,20 & 9,20 \\
\hline 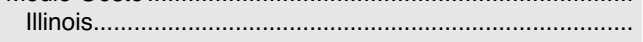 & 4,40 & 4,00 & 4,30 & 4,00 & 3,90 \\
\hline
\end{tabular}

Las instituciones educativas, conscientes de la necesidad de adaptar sus contenidos curriculares a esta realidad empresarial, han comenzado a ofrecer a sus alumnos distintas opciones para estudiar negocios en español. La creciente demanda de este tipo de cursos plantea la necesidad de mejorar su proceso de enseñanza. Ese es precisamente el objetivo que se pretende alcanzar con este artículo que, tras analizar el estado actual de la cuestión, identifica las mejores estrategias educativas que, convenientemente promovidas y mejoradas, permitirán perfeccionar la enseñanza de esta materia.

Tras analizar una muestra de las 100 mejores facultades de empresariales/escuelas de negocios de EEUU, se extrae como primera conclusión que, a pesar de que la comunidad hispanohablante se concentra fundamentalmente en los estados de California y Texas (Tabla 4), todas las instituciones consultadas, distribuidas por la totalidad de la geografía estadounidense, ofrecen la posibilidad de combinar el estudio del español con los temas empresariales.

Ahora bien, los niveles de integración de ambas materias varían de forma significativa con un primer nivel que engloba aquellas universidades que, si bien no brindan la posibilidad de estudiar negocios en español, ofrecen asignaturas de empresariales e idiomas por separado. En el segundo nivel encontraríamos las 52 universidades que combinan el estudio de ambas materias a través de cursos como «Español para los Negocios». A pesar de que esta sea la asignatura que se imparte con mayor frecuencia, también se ofrecen otros cursos que se centran en aspectos de carácter más social y cultural. Finalmente, en el tercer nivel, se encontrarían los 34 centros que ofrecen titulaciones específicas que permiten al alumno adquirir una formación más integral sobre Español para los Negocios. Las opciones de esta categoría varían desde especialidades lingüísticas ofrecidas por los departamentos de idiomas hasta títulos de grado, y posgrado y cursos de formación continua profesional incluidos en los catálogos de las facultades de empresariales y escuelas de negocios.

El análisis realizado ha permitido identificar una gran multitud de estrategias que pueden mejorar y promover la enseñanza del Español para los $\mathrm{Ne}$ gocios. Entre ellas destacan la formación práctica, las estancias internacionales, la flexibilidad curricular, el enfoque multidisciplinar, el uso de las TIC y la promoción de la multiculturalidad, es decir, de la diversidad tanto de alumnos como de profesores. Estas estrategias educativas, convenientemente aplicadas y si es posible mejoradas, contribuirán a consolidar al español como uno de los idiomas con mayor peso en el mundo empresarial del siglo XXI.

\section{Bibliografía}

[1] BLOOMBERG BUSINESSWEEK (2015). Disponible en:

http://www.businessweek.com/bschools/ rankings\#5 
[2] FORBES INSIGHTS (2011). Global Diversity and Inclusion: Fostering Innovation through a Diverse Workforce. Disponible en:

http://images. forbes.com/forbesinsights/StudyPDFs /Innovation_Through_Diversity.pdf

[3] GEOSPACE (2014). Hispanic Businesses \& Entrepreneurs Drive Growth in the New Economy. $2^{\text {nd }}$ Annual Report 2014. Disponible en: http://www.geoscape.com/HBR/pdf/Geoscape_ HispanicBusinessOwners_FINAL.pdf

[4] GURIN, P.; NAGDA, B.A. y LÓPEZ, G.E. (2004). «The Benefits of Diversity in Education for Democratic Citizenship». Journal of Social Issues, $\mathrm{n}^{\circ}$ 60, pp. 17-34.

[5] ICEX ESPAÑA EXPORTACIÓN E INVERSIONES (2014). Informe de Empresas Españolas establecidas en EEUU - Estados del Noreste (03-marzo-2014). Disponible en:

http://www.icex.es/icex/es/navegacion-principal/ implantacion-e-inversion-exterior/informacionpara-invertir-en-el-exterior/empresas-espanolas-establecidas-en-el-exterior/4646579.html

[6] INSTITUTO CERVANTES (2014). El español en el mundo. Anuario del Instituto Cervantes 2014. Madrid: Imprenta Nacional de la Agencia Estatal Boletín Oficial del Estado.

[7] INSTITUTO NACIONAL DE ESTADÍSTICA (2015). Disponible en: http://ine.es/
[8] JIMÉNEZ, J.C. y NARBONA, A. (2012). El español en los flujos económicos internacionales. Barcelona: Ariel.

[9] KITCHENER, K.; WOOD, P. y JENSEN, L. (2000, August). «Curricular, co-curricular, and institutional influence on real-world problem-solving». Paper presented at the annual meeting of the American Psychological Association, Boston, MA.

[10] OFICINA DEL CENSO DE LOS ESTADOS UNIDOS (2010). 2010 Census Data. Disponible en: www.census.gov/2010census/data

[11] PASCARELLA, E.; PALMER, B.; MOYE, M. y PIERSON, C. (2001). «Do diversity experiences influence the development of critical thinking?» Journal of College Student Development, $n^{\circ} 42$, pp. 257-291.

[12] PEW RESEARCH CENTER (2015). Disponible en: http://www.pewresearch.org/

[13] SELIG CENTER FOR ECONOMIC GROWTH (2014). Buying Power: The Multicultural Economy 2014. The Selig Center for Economic Growth, Terry College of Business, University of Georgia, GA.

[14] THOMSON, A. y CUSEO, J.B. (2014). Diversity and the College Experience: Research-based strategies for appreciating human differences. Kendall/ Hunt Publishing Company, IA. 


\section{Virginia Cortijo}

ANEXO

\section{Universidades de la muestra}

\begin{tabular}{|c|c|c|c|c|}
\hline $\begin{array}{c}\text { Ranking } \\
2014\end{array}$ & Universidades & Alumnos matriculados & Estado & Zona \\
\hline 1 & University of Notre Dame & 1.968 & Indiana, IN & D3 \\
\hline 2 & University of Virginia & 679 & Virginia, VA & D5 \\
\hline 3 & Cornell University & 737 & New York, NY & $\mathrm{D} 2$ \\
\hline 4 & Boston College & 2.004 & Massachusetts, MA & D1 \\
\hline 5 & Washington University in St. Louis & 902 & Missouri, MO & D4 \\
\hline 6 & University of Texas, Austin & 4.046 & Texas, TX & D7 \\
\hline 7 & University of Pennsylvania & 2.502 & Pennsylvania, PA & $\mathrm{D} 2$ \\
\hline 8 & Indiana University & 5.001 & Indiana, IN & D3 \\
\hline 9 & Emory University & 742 & Georgia, GA & D5 \\
\hline 10 & University of North Carolina, Chapel Hill & 676 & North Carolina, NC & D5 \\
\hline 11 & Wake Forest University & 538 & North Carolina, NC & D5 \\
\hline 12 & University of Michigan, Ann Arbor & 1.421 & Michigan, MI & D3 \\
\hline 13 & Brigham Young University & 1.542 & Utah, UT & D8 \\
\hline 14 & New York University & 2.495 & New York, NY & D2 \\
\hline 15 & University of California, Berkeley & 717 & California, CA & D9 \\
\hline 16 & University of Richmond & 477 & Virginia, VA & D5 \\
\hline 17 & Carnegie Mellon University & 379 & Pennsylvania, PA & D2 \\
\hline 18 & Georgetown University & 1.298 & Washington, DC & D5 \\
\hline 19 & Northeastern University & 3.712 & Massachusetts, MA & D1 \\
\hline 20 & Bentley University & 4.056 & Massachusetts, MA & D1 \\
\hline 21 & Southern Methodist University & 1.193 & Texas, TX & D7 \\
\hline 22 & The College of William \& Mary & 502 & Virginia, VA & D5 \\
\hline 23 & Miami University & 2.779 & Ohio, $\mathrm{OH}$ & D3 \\
\hline 24 & Villanova University & 1.602 & Pennsylvania, PA & D2 \\
\hline 25 & Boston University & 2.400 & Massachusetts, MA & D1 \\
\hline 26 & Babson College & 2.106 & Massachusetts, MA & D1 \\
\hline 27 & Texas Christian University & 1.918 & Texas, TX & D7 \\
\hline 28 & University of Southern California & 3.825 & California, CA & D9 \\
\hline 29 & Texas A\&M University & 4.692 & Texas, TX & D7 \\
\hline 30 & The Pennsylvania State University & 5.405 & Pennsylvania, PA & $\mathrm{D} 2$ \\
\hline 31 & Lehigh University & 1.343 & Pennsylvania, PA & D2 \\
\hline 32 & The Ohio State University & 3.770 & Ohio, $\mathrm{OH}$ & D3 \\
\hline 33 & University of Wisconsin-Madison & 1.990 & Wisconsin, WI & D3 \\
\hline 34 & University of Illinois, Urbana-Champaign & 2.948 & Illinois, IL & D3 \\
\hline 35 & Rensselaer Polytechnic Institute & 307 & New York, NY & D2 \\
\hline 36 & University of Massachusetts, Amherst & 2.369 & Massachusetts, MA & D1 \\
\hline 37 & Georgia Institute of Technology & 1.242 & Georgia, GA & D5 \\
\hline 38 & Fordham University & 1.953 & New York, NY & D2 \\
\hline 39 & University of Maryland & 2.696 & Maryland, MD & D5 \\
\hline 40 & James Madison University & 3.273 & Virginia, VA & D5 \\
\hline 41 & University of San Diego & 1.158 & California, CA & D9 \\
\hline 42 & Michigan State University & 1.890 & Michigan, MI & D3 \\
\hline 43 & Santa Clara University & 1.405 & California, CA & D9 \\
\hline 44 & University of Florida & 1.444 & Florida, FL & D5 \\
\hline 45 & Elon University & 1.625 & North Carolina, NC & D5 \\
\hline 46 & University of Minnesota & 2.280 & Minnesota, MN & D4 \\
\hline 47 & Loyola University Maryland & 1.049 & Maryland, MD & D5 \\
\hline 48 & University of Georgia & 2.345 & Georgia, GA & D5 \\
\hline 49 & Bryant University & 2.190 & Rhode Island, RI & D1 \\
\hline 50 & Case Western Reserve University & 330 & Ohio, $\mathrm{OH}$ & D3 \\
\hline 51 & Butler University & 881 & Indiana, IN & D3 \\
\hline 52 & University of Washington & 1.635 & Washington, WA & D9 \\
\hline 53 & University of Miami & 2.115 & Florida, FL & D5 \\
\hline
\end{tabular}


ANEXO (continuación) Universidades de la muestra

\begin{tabular}{|c|c|c|c|c|}
\hline $\begin{array}{c}\text { Ranking } \\
2014\end{array}$ & Universidades & Alumnos matriculados & Estado & Zona \\
\hline 54 & Binghamton University & 1.473 & New York, NY & D2 \\
\hline 55 & Syracuse University & 1.835 & New York, NY & D2 \\
\hline 56 & University of Texas, Dallas & 2.371 & Texas, TX & D7 \\
\hline 57 & Purdue University & 2.469 & Indiana, IN & D3 \\
\hline 58 & Fairfield University & 1.231 & Connecticut, CT & D1 \\
\hline 59 & George Washington University & 1.530 & Washington, DC & D5 \\
\hline 60 & Chapman University & 1.338 & California, CA & D9 \\
\hline 61 & Seattle University & 959 & Washington, WA & D9 \\
\hline 62 & American University & 1.002 & Washington, DC & D5 \\
\hline 63 & The College of New Jersey & 1.145 & New Jersey, NJ & D2 \\
\hline 64 & DePaul University & 3.850 & Chicago, IL & D3 \\
\hline 65 & University of Connecticut & 1.729 & Connecticut, CT & D1 \\
\hline 66 & Quinnipiac University & 1.597 & Connecticut, CT & D1 \\
\hline 67 & University of Denver & 1.112 & Colorado, $\mathrm{CO}$ & D8 \\
\hline 68 & Baylor University & 3.160 & Texas, $\mathrm{TX}$ & D7 \\
\hline 69 & Virginia Polytechnic Institute \& State University & 3.669 & Virginia, VA & D5 \\
\hline 70 & California Polytechnic State University & 2.416 & California, CA & D9 \\
\hline 71 & University of Arizona & 1.434 & Arizona, AZ & D8 \\
\hline 72 & Ohio Northern University & 291 & Ohio, $\mathrm{OH}$ & D3 \\
\hline 73 & Colorado State University & 2.106 & Colorado, CO & D8 \\
\hline 74 & Tulane University & 1.718 & Louisiana, LA & D7 \\
\hline 75 & Providence College & 1.225 & Rhode Island, RI & D1 \\
\hline 76 & University of Alabama & 6.894 & Alabama, AL & D6 \\
\hline 77 & John Carroll University & 392 & Ohio, $\mathrm{OH}$ & D3 \\
\hline 78 & Seton Hall University & 824 & New Jersey, NJ & $\mathrm{D} 2$ \\
\hline 79 & Ohio University & 1.786 & Ohio, $\mathrm{OH}$ & D3 \\
\hline 80 & Marquette University & 1.709 & Wisconsin, WI & D3 \\
\hline 81 & University of Tulsa & 805 & Oklahoma, OK & D7 \\
\hline 82 & Loyola Marymount University & 1.469 & California, CA & D9 \\
\hline 83 & Texas Tech University & 3.700 & Texas, TX & D7 \\
\hline 84 & University of Pittsburgh & 2.092 & Pennsylvania, PA & D2 \\
\hline 85 & Florida State University & 5.240 & Florida, FL & D5 \\
\hline 86 & North Carolina State University & 2.391 & North Carolina, NC & D5 \\
\hline 87 & Sacred Heart University & 813 & Connecticut, CT & D1 \\
\hline 88 & University of Missouri & 4.252 & Missouri, MO & D4 \\
\hline 89 & St. Joseph's University & 2.325 & Pennsylvania, PA & D2 \\
\hline 90 & Bowling Green State University & 574 & Ohio, $\mathrm{OH}$ & D3 \\
\hline 91 & Rochester Institute of Technology & 647 & New York, NY & D2 \\
\hline 92 & Arizona State University & 4.523 & Arizona, AZ & D8 \\
\hline 93 & Belmont University & 1.735 & Tennessee, TN & D6 \\
\hline 94 & University of lowa & 2.029 & lowa, IA & D4 \\
\hline 95 & Illinois State University & 2.997 & Illinois, IL & D3 \\
\hline 96 & University of Delaware & 2.822 & Delaware, DE & D5 \\
\hline 97 & University of Colorado at Boulder & 2.866 & Colorado, CO & D8 \\
\hline 98 & St. Louis University & 1.373 & Missouri, MO & D4 \\
\hline 99 & University of Houston & 1.930 & Texas, TX & D7 \\
\hline 100 & University of Cincinnati & 3.114 & Ohio, $\mathrm{OH}$ & D3 \\
\hline
\end{tabular}


